Pierre-Alain Duc, Jonathan Braine and Elias Brinks, eds.

\title{
HI and Hot Gas in the Outskirts of the M81 Group
}

\author{
M. Bureau \\ Hubble Fellow, Columbia Astrophysics Laboratory, 550 West 120th \\ Street, 1027 Pupin Hall, Mail Code 5247, New York, NY 10027, U.S.A. \\ F. Walter \\ Jansky Fellow, NRAO, P.O. Box O, Socorro, NM 87801, U.S.A.
}

\section{J. van Gorkom}

Department of Astronomy, Columbia University, 538 West 120th Street, New York, NY 10027, U.S.A.

\section{Carignan}

Département de Physique, Université de Montréal, C.P. 6128, Succ. Centre-Ville, Montréal, QC H3C 3J\%, Canada

\begin{abstract}
Results are presented from a wide area, high resolution HI synthesis survey of the outer regions of the nearby M81 group, where internal (galactic) and external (group-related) evolution processes can be studied simultaneously in great detail. The survey encompasses the star forming dwarf galaxies M81dwA, UGC4483, and HoII, where evidence of ram pressure stripping was recently discovered. The data do not reveal any intergalactic HI, but the outer parts of HoII are reminiscent of tidal tails. We argue however that those structures are equally consistent with the latest ram pressure models including cooling. The case for a hot intergalactic medium in this poor, spiral-only group is thus still open. The survey also puts tight constraints on possible counterparts to the local high velocity cloud population in an external group, reaching a $3 \sigma$ column density of $10^{19}$ atom $\mathrm{cm}^{-2}$ and a $6 \sigma$ limiting mass of $1.5 \times 10^{5} \mathrm{M}_{\odot}$.
\end{abstract}

\section{Introduction}

Most galaxies in the local universe are located in groups, where dwarfs dominate by number. Understanding the influence of this environment on (dwarf) members is therefore essential to our comprehension of galaxy formation and evolution. Nearby groups are particularly attractive for this purpose since both internal (galactic) and external (group-related) evolution processes can be studied in detail. Bureau \& Carignan (2002) presented neutral hydrogen Very Large Array (VLA) D-array observations of the dwarf irregular galaxy HoII, a prototype galaxy for studies of self-propagating star formation (Puche et al. 1992). HI was detected to radii over $16^{\prime}$ or $4 R_{25}$, with a total mass $M_{\mathrm{HI}}=6.44 \times 10^{8} M_{\odot}$. 
Most importantly, the integrated HI map revealed a characteristic comet-like appearance, with a large but faint component extending to the northwest and the HI appearing compressed on the opposite side, suggesting that HoII is affected by ram pressure from an intragroup medium (IGM).

HoII lies roughly $0.5 \mathrm{Mpc}$ northeast of the core of the nearby M81 Group $(D=3.2 \mathrm{Mpc})$, along with the dwarf irregular galaxy M81dwA (Kar52) and the blue compact dwarf galaxy UGC4483. No obvious signs of interaction were detected, but the three galaxies are most likely part of the NGC2403 subgroup, infalling toward M81 (Karachentsev et al. 2000). With large uncertainties, ram pressure stripping of the outer parts of the disk would require an IGM density $n_{\mathrm{IGM}} \gtrsim 4.0 \times 10^{-6}$ atoms $\mathrm{cm}^{-3}$ near HoII, or about $1 \%$ of the virial mass of the group uniformly distributed over a volume just enclosing it, consistent with the known X-ray properties of small groups. The presence of an IGM in such a poor group, lacking any early-type galaxy, would have important consequences for the evolution of group members, and it could explain why many HI holes in HoII are located in low surface density regions of the disk, where no star formation is expected or observed (Rhode et al. 1999; Bureau \& Carignan 2002).

\section{Survey}

To test whether the HI morphology of HoII is indeed due to ram pressure stripping, and thus to the existence of an extended hot IGM, or whether tidal interactions offer a better explanation, we have conducted an HI survey of the area encompassing HoII, M81dwA, and UGC4483. Our setup is entirely driven by the need for excellent surface brightness sensitivity, and constraints on the presence of any intergalactic HI clouds are obtained for free.

A mosaic of 36 contiguous VLA D-array pointings was obtained over 5 separate observing runs, each pointing totaling roughly $50 \mathrm{~min}$ of integration on source. The mosaic partially covers an area of about $3^{\circ} \times 3^{\circ}$, with a pointing spacing of $22^{\prime}$, ensuring proper sampling of the $32^{\prime}$ primary beam. A $3.125 \mathrm{MHz}$ bandwidth with 64 channels was used for all observations $\left(10.3 \mathrm{~km} \mathrm{~s}^{-1}\right.$ per channel; online Hanning smoothing), covering the entire velocity range of the three galaxies and the M81 and NGC2403 groups, while simultaneously resolving the internal kinematics of individual galaxies. Except at the edges, the rms noise per channel achieved is approximately uniform at $1.0 \mathrm{mJy}^{\text {beam }}{ }^{-1}$, corresponding to $3.0 \times 10^{18}$ atoms $\mathrm{cm}^{-2}$ or $2.5 \times 10^{4} M_{\odot}$ at the distance adopted. Preliminary total HI and intensity-weighted mean velocity fields of the entire mosaic are presented in Figure 1, while blow-ups of the HoII-M81dwA pair are shown in Figures 2 and 3. The synthesized beam is $65^{\prime \prime} \times 65^{\prime \prime}$ in all maps.

\section{Large-Scale Structure and Intergalactic HI}

The main result from the large-scale HI structure presented in Figure 1 is that no $\mathrm{HI}$ is detected outside that directly associated with HoII, M81dwA, and UGC4483. That is, there is no evidence of either large-scale tidal interactions or any intergalactic HI cloud population within a broad area encompassing the three galaxies (and more). 

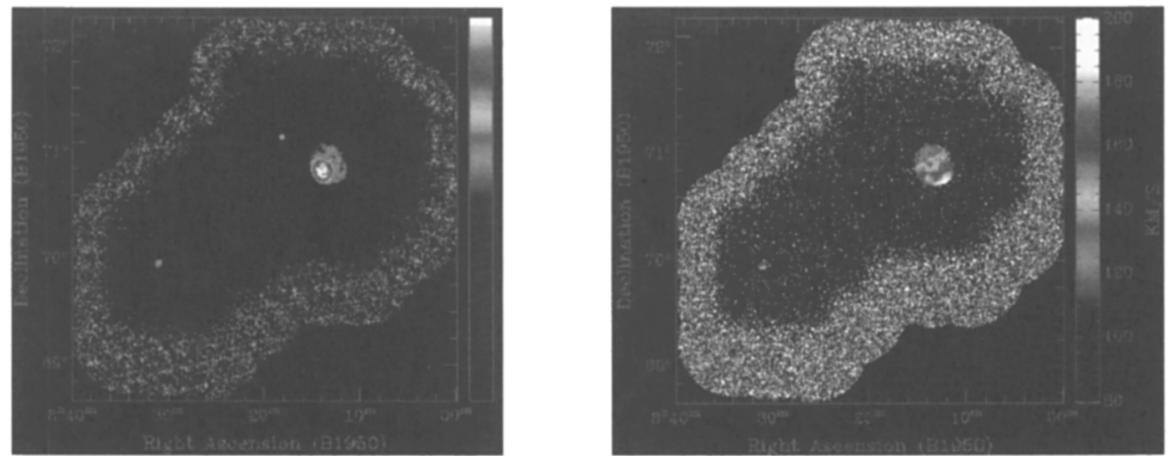

Figure 1. Left: Total HI map of the entire survey area (logarithmic fluxes). Note the absence of large-scale HI beside that associated with the three galaxies. Right: Intensity-weighted mean velocity field.

Our excellent surface brightness sensitivity ensures that we can detect intergalactic HI down to column densities of about $1.0 \times 10^{19}$ atoms $\mathrm{cm}^{-2}$ at the $3 \sigma$ level, below which the hydrogen is probably ionized by the metagalactic UV radiation field. Across the entire mosaic, no $\mathrm{HI}$ is detected in any channel at a level of $5 \sigma$, corresponding to $1.5 \times 10^{19}$ atoms cm $\mathrm{cm}^{-2}$ or $1.25 \times 10^{5} M_{\odot}$ (per beam, of $1 \mathrm{kpc}$ diameter at the distance adopted). Although we have not yet carried out a proper statistical analysis, no patch of material at a lower column density is detected either, so tighter limits apply to extended material and limits on the volume density of various intergalactic HI cloud populations will be derived.

Those limits are extremely interesting considering the current debate about the nature of the high velocity clouds (HVCs) surrounding the Galaxy. Blitz et al. (1999) suggest that some HVCs are the long sought minihalos overproduced in cold dark matter structure formation simulations. A typical compact HVC has a mass of $4.5\left(\frac{D}{\mathrm{kpc}}\right)^{2} \times 10^{6} M_{\odot}$ and a size of $0.4 \mathrm{deg}^{2}$, corresponding to about $1 \times 10^{6} M_{\odot}$ and a diameter of $6 \mathrm{kpc}$ at the typical expected distance of $500 \mathrm{kpc}$ $\left(N_{\mathrm{HI}}=1 \times 10^{19}\right.$ atoms $\mathrm{cm}^{-2}$ at peak and $\sigma_{\mathrm{V}}=25 \mathrm{~km} \mathrm{~s}^{-1}$; Putman et al. 2002). If present, counterparts to the Local Group HVCs should thus appear as large patches of faint HI in our data (many beam widths). A cursory examination of our cube reveals no such signal, so the analogs of Local Group HVCs remain undetected in the M81 Group if located at hundreds of kiloparsecs from the core (where our observations are located and the limits become interesting). It remains to be seen however if the low surface brightness material in the northwest half of HoII would be seen as HVCs by an observer located within its disk.

\section{HoII and M81dwA}

Given the absence of large-scale HI or intergalactic clouds, we now focus our attention on the HoII-M81dwA pair (Figs. 2-3). HoII itself still shows a high surface brightness component which appears axisymmetric (except for the presence of numerous holes and shells) and is clearly rotationally supported. The sharp edge to the southwest of the HI distribution is also very much present. 


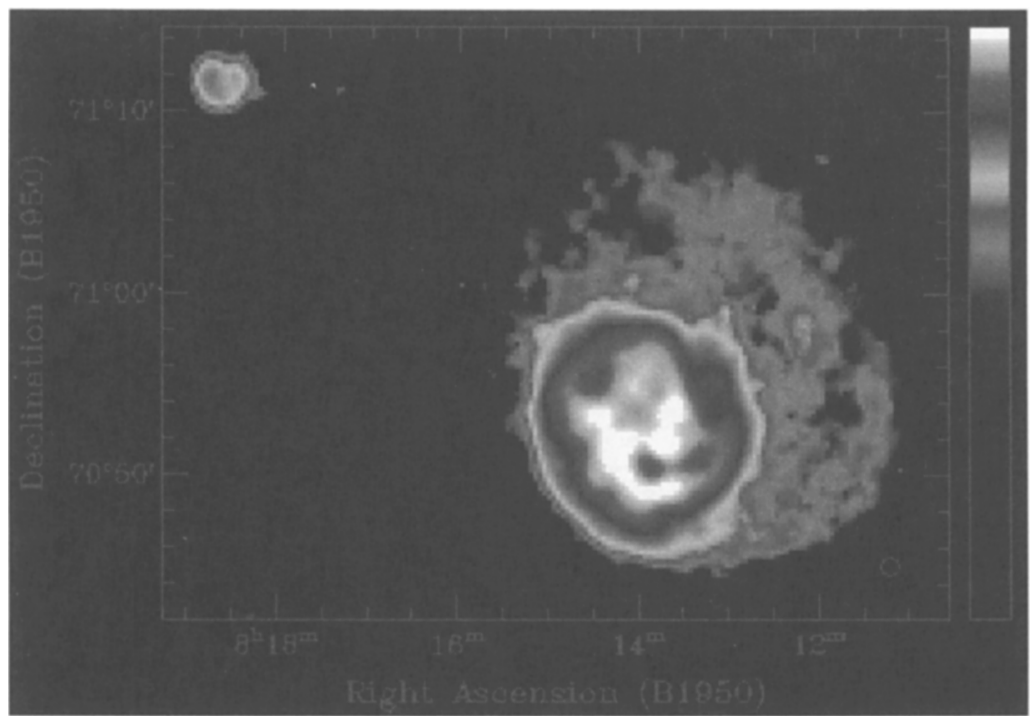

Figure 2. Total HI map of the survey area near HoII and M81dwA (logarithmic fluxes).

The low surface brightness component to the northwest, which except for its comet-like morphology appeared structureless in the shallower data of Bureau \& Carignan (2002), is now resolved into at least two and perhaps three arms. In particular, the arm to the southwest can be traced through over $\frac{1}{2}$ turns, and perhaps $1 \frac{1}{2}$, in the channel maps.

HoII's HI morphology is reminiscent of that expected from tidal interactions, M81dwA providing a likely interacting companion. The arm kinematics is however regular, M81dwA appears undisturbed, and no bridge is visible between the two galaxies (or any debris outside of their immediate vicinities). The case for a tidal interaction at the origin of HoII's HI distribution and kinematics is thus not bullet-proof, and the possibility of ram pressure stripping can not be rejected outright. We explore each of those possibilities in more details below.

\subsection{Tidal Interactions}

The HI distribution of HoII displays some of the characteristics expected from the interaction of a disk galaxy with a passing lower mass companion: strong asymmetry, sharp "near" tidal arm, and broader "far" tidal arm. In such cases, the arms are material, as appears to be the case here. The argument for an HoII-M81dwA tidal interaction can be enlightened by a comparison with the well-studied case of M51 and its companion NGC5195. In M51, the inner spiral arms were probably caused by a close passage of NGC5195 (on a bound orbit), while the outer tidal arm was most likely caused by a farther and earlier passage (e.g. Howard \& Byrd 1990). The latter in particular is very similar to the morphology of HoII, located well outside of the optical body of the galaxy and composed essentially entirely of HI (Rots et al. 1990). It is extended, one-sided, curved, with a sharp edge on its leading side and clumps of HI at its end. 


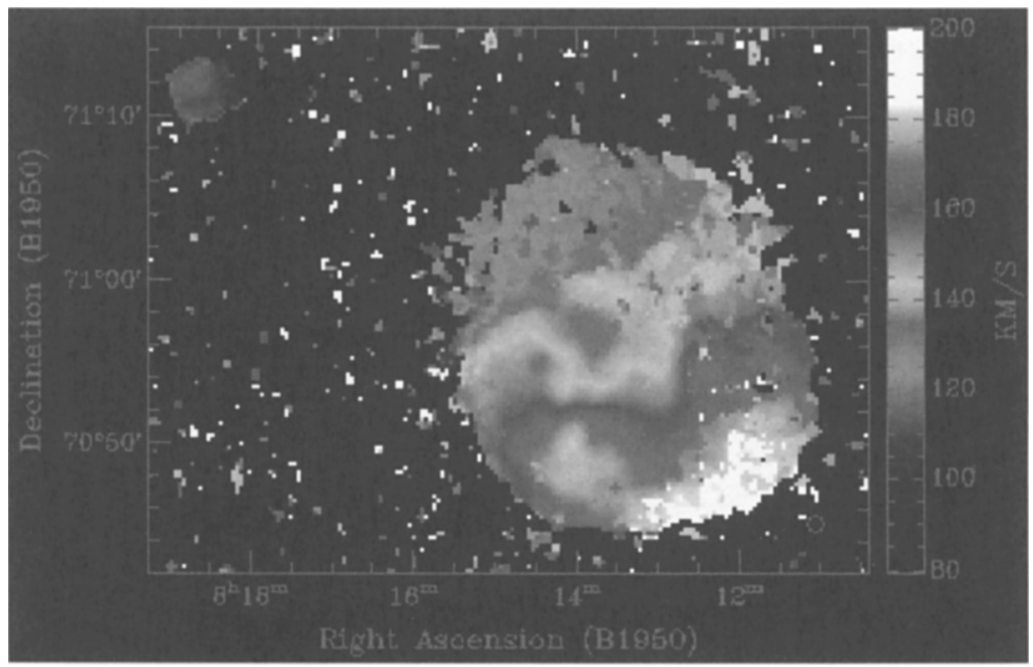

Figure 3. Intensity-weighted mean HI velocity field of the survey area near HoII and M81dwA.

The HI clumps at the end of M51's tidal arm are also interesting in that they are located roughly where M81dwA currently lies with respect to HoII, and they are expected in a tidal model (Howard \& Byrd 1990). This raises the possibility that M81dwA is not the companion at the origin of the disturbed morphology of HoII, but rather a tidal dwarf, i.e. a low-mass galaxy formed through gravitational collapse of the tidal debris from a previous interactions, perhaps with UGC4483. This possibility is supported by the kinematics of M81dwA, which appears to contain little if any dark matter (Sargent, Sancisi, \& Lo 1983), but it must remain speculative until detailed modeling of the HoIIM81dwA pair (and possibly of all three galaxies) is carried out.

\subsection{Ram Pressure Stripping}

The arms detected within the northwest low surface brightness HI component of HoII are not normally associated with ram pressure, which tends to create clumpy and disorganized comet-like tails (e.g. Quilis, Moore, \& Bower 2000). However, recent models including gas cooling do yield similar structures (Schulz \& Struck 2001). As usual, a face-on galaxy-IGM encounter yields a symmetric gas distribution, while an inclined encounter yields an asymmetric one. The latter is indeed expected in the case of HoII given its location with respect to the core of the M81 Group. Most importantly, when the compressed gas (due to ram pressure) is allowed to cool, it becomes gravitationally unstable, develops spiral arms, and loses angular momentum to the outer disk, thus shrinking in the process. This process of compression-driven annealing also leads to the formation of a sharp edge and a dense annulus in the inner parts of the galaxy, in effect shielding the center of the galaxy from further stripping, while abandoning the outer disk to the IGM (Schulz \& Struck 2001). The resulting morphology is very much like that observed in HoII (Fig. 2). 
Given the coupling between the disk rotation and the IGM "wind" in ram pressure simulations including cooling, it is not possible to reject outright the possibility that ram pressure is indeed at the origin of HoII's HI morphology.

\section{Conclusions}

We have presented preliminary results from a wide-field but sensitive HI synthesis survey of the outskirts of the M81 Group, in an area encompassing the dwarf galaxies HoII, M81dwA, and UGC4483. No signs of large-scale tidal features are present, and no intergalactic HI clouds are detected, this to a level better than $1.5 \times 10^{19}$ atoms $\mathrm{cm}^{-2}$ or $1.25 \times 10^{5} M_{\odot}$ (per channel and beam), which appears to rule out counterparts to the Local Group HVCs if located at cosmologically relevant distances. The HI distribution and kinematics of HoII are consistent with that expected from tidal interactions, especially M51-like events, but ram pressure models including gas cooling are also possible. It thus appears premature to rule out either possibility, and thus the presence of a hot extended IGM in the M81 Group. It is also possible that M81dwA is a tidal dwarf. A search for a (faint) stellar counterpart to the northwest HI arms in HoII should resolve the issue, given that ram pressure is only expected to affect the gas.

Acknowledgments. Support for this work was provided by NASA through Hubble Fellowship grant HST-HF-01136.01 awarded by the Space Telescope Science Institute, which is operated by the Association of Universities for Research in Astronomy Inc. for NASA under contract NAS 5-26555, and by NSF grant AST 00-98249 to Columbia University. The Digitized Sky Surveys were produced at the Space Telescope Science Institute under U.S. Government grant NAG W-2166. The images of these surveys are based on photographic data obtained using the Oschin Schmidt Telescope on Palomar Mountain and the UK Schmidt Telescope. The plates were processed into the present compressed digital form with the permission of these institutions.

\section{References}

Blitz, L., Spergel, D. N., Teuben, P. J., Hartmann, D., \& Burton, W. B. 1999, ApJ, 514, 818

Bureau, M., \& Carignan, C. 2002, AJ, 123, 1316

Howard, S., \& Byrd, G. G. 1990, AJ, 99, 1798

Karachentsev, I. D., et al. 2000, A\&A, 363, 117

Puche, D., Westpfahl, D., Brinks, E., \& Roy, J.-R. 1992, AJ, 103, 1841

Putman, M. E., et al. 2002, AJ, 123, 873

Quilis, V., Moore, B., \& Bower, R. 2000, Science, 288, 1617

Rhode, K. L., Salzer, J. J., Westpfahl, D. J., \& Radice, L. A. 1999, AJ, 118, 323

Rots, A. H., Crane, P. C., Bosma, A., Athanassoula, E., \& van der Hulst, J. M. 1990, AJ, 100, 387

Sargent, W. L. W., Sancisi, R., \& Lo, K. Y. 1983, ApJ, 265, 711

Schulz, S., \& Struck, C. 2001, MNRAS, 328, 185 